\title{
APPROCHE DES CRIS PHILOSOPHIQUES. FORMES DE PENSÉES SINGULIÉRES DANS LES PRATIQUES PHILOSOPHIQUES AVEC LES ENFANTS
}

\begin{abstract}
ANDA FOURNEL ${ }^{1}$
ABSTRACT. The discursive and reflexive practices falling within "Philosophy for children" (P4C) program aim to enable children to develop autonomous and authentic thinking by transforming the class into a community of philosophical inquiry. The educational program's initiators put forward the idea that learning to think cannot exclusively follow a rationality model. Considering Deleuze distinction (1984) between "cry of reason" and "cry of unreason" as a starting point, we are interested in cry/s specimens - the concept and the question - as the call's living forms in philosophical thought, linked to the emotions' expression. Our investigation explores the folds of children's thinking, through a qualitative analyze of classroom recordings in primary and middle school, in order to asses children's ability to provide "philosophical cries". If so, one might wonder what do they shout and how do they do it?
\end{abstract}

Keywords: community of philosophical inquiry — philosophical cry — emotions concept - question.

RÉSUMÉ. Les pratiques discursives et réflexives promues par le programme " Philosophy for Children » (P4C) ont pour objectif de permettre aux enfants de développer une pensée autonome et authentique, par la transformation de la classe en une communauté de recherche philosophique. Les initiateurs de ce programme avancent l'idée qu'apprendre à penser ne peut pas suivre exclusivement un modéle de rationalité. En prenant comme point de départ la distinction proposée par Deleuze (1984) entre « cri de raison » et « cri de déraison ", je m'intéresse à des (proto)types de " cris » - des concepts et des questions -

\footnotetext{
${ }^{1}$ Docteure en Sciences du Langage de I'Université Grenoble Alpes, diplômée en Philosophie (Roumanie) et en Sciences de l'éducation (France). Ses recherches portent notamment sur les pratiques philosophiques avec les enfants, dans une perspective pragmatique. Courriel : anda.fournel@univ-grenoble-alpes.fr.
} 
en tant que formes vivantes d'interpellation dans une pensée philosophique, liés à l'expression des émotions. Mon investigation sillonne les plis de la pensée des enfants, travers des analyses qualitatives d'enregistrements réalisées en école primaire et au collège, afin d'observer si les enfants sont capables ou non de crier philosophiquement. Si oui, qu'est-ce qu'ils ont à crier et comment le font-ils ?

Mots-clés : communauté de recherche philosophique - cri philosophique émotions - concept - question.

Si nous tenons à un minimum d'équilibre, remettons-nous au cri, ne perdons aucune occasion de nous y jeter et d'en proclamer l'urgence. (Emil Cioran, La chute dans le temps, 1964)

\section{L'émotion en philosophie}

Tout le monde peut philosopher mais encore faut-il savoir le faire. La compétence philosophique s'apprend, au point qu'elle peut devenir une méthode. Mais qu'en est-il de l'empirisme d'une pensée philosophique, de sa force primaire et incarnée, de son acuité et son caractère inattendu, de sa spontanéité ?

Il existe, parmi les philosophes, ceux qui s'expriment avec l'urgence des cris — signe le plus souvent de lucidité - pour attirer l'attention sur ce qui leur semble essentiel. Le verbe, en latin classique, quǐrĩto, quǐrĩtãre (critare, en latin populaire) ${ }^{2}$ signifie " appeler au secours ", " protester à grands cris " et renvoie donc à l'intention d'un auteur d'alerter, d'avertir ou de mettre en garde ses semblables sur la présence d'un danger, l'urgence d'une information, l'impératif d'une idée. Pousser un cri se fait " sous l'effet d'une émotion, d'un état physique ou moral ressenti intensément $\|^{3}$. L'image du philosophe proposé par Platon dans l'allégorie de la caverne est celle d'une personne qui choisit de vivre dans la vérité ; sa mission est alors d'avertir ceux qui se laissent prendre au piège de l'illusion et vivent prisonniers de leurs jugements, fausses idées reçues ou croyances, pour les amener à la lumière. Lorsque cette émotion prend une dimension métaphysique, chez des auteurs comme Cioran, le cri s'apparente à une raison d'être et délimite comme un espace de vie.

Exception faite notamment pour la pensée hellénistique, on a souvent reproché à la philosophie de s'éloigner de la vie, de se protéger des émotions et de se consacrer plutôt à la recherche des conditions de la connaissance et à la pure réflexion. Pourtant, séparer la démarche philosophique d'une quête de sens ou d'une manière de vivre, d'une construction de soi ou d'un engagement éthique ou politique est impensable. Prenons le doute et le questionnement qui participent de

\footnotetext{
${ }^{2}$ Trésor de la Langue Française informatisé (TLFI), art. « cri » :

http://atilf.atilf.fr/dendien/scripts/tlfiv5/visusel.exe?11;s=3342396945;r=1;nat=;sol=0; 3 Ibid.
} 
la problématisation philosophique. Que seraient-ils sans la prise de conscience d'un problème incarné par un étonnement, une indignation, une intuition : Comment est-ce possible ? Je ne comprends pas! Je perçois un problème. Des auteurs comme Popper (1960) et, bien avant, Peirce (1877), parlent de malaise, d'inconfort, de mécontentement ou d'irritation épistémiques qui seraient à l'origine de toute démarche de recherche ou d'action. Ces états d'âme traduiraient l'engagement subjectif du questionneur, mû par un désir d'atteindre un état de savoir ou de calme psychologique (Peirce l'appelle " croyance »).

C'est un constat : les émotions, les affects et la dimension corporelle de la pensée ont été très peu valorisés en philosophie. L'intérêt porté aux émotions dans I'histoire des idées apparaît tôt, et principalement à travers le souci de régulariser l'énergie affective. Les philosophes de l'Antiquité conseillaient d'en faire bon usage. Soupçonnées de perturber le fonctionnement optimal de la raison, les émotions étaient souvent mises en opposition avec la raison. Descartes (1990) opposait les « émotions intellectuelles ", contrôlées par la volonté, aux " émotions corporelles ", envisagées comme des mouvements «non contrôlés ». Il s'avérait nécessaire, dans la vision du penseur rationaliste, de se mobiliser intellectuellement contre les mouvements désordonnés de l'âme et de mettre à distance ses émotions. Les empiristes admettront, au contraire, que les émotions jouent un rôle déterminant dans la production des jugements moraux (Hume, 1739/1993, III, I, I, 52-53). Avec la philosophie contemporaine, Sartre (1938/1995) et récemment Nussbaum (2004), par exemple, mettent en avant la dimension " évaluative " des émotions. On doit notamment au livre de Damasio (2010) - L'Erreur de Descartes, la revalorisation des émotions sur le plan cognitif, l'auteur s'attachant à montrer qu'il est possible d'être rationnel sans se couper de ses émotions. Enfin, les approches incarnées et dynamiques de la cognition (Varela, Thompson \& Rosch, 1991) et les propositions phénoménologiques autour de la subjectivité et de l'expérience humaine vont également dans ce sens.

La rationalité et le caractère abstrait du concept ont été par ailleurs également mis en question. Le concept est avant tout l'œuvre de l'intellect qui organise le monde et la diversité du réel grâce à des opérations mentales telles qu'ordonner, classer, généraliser, etc. La tradition rationaliste opposait deux modes de connaissance : l'intuition, qui porte directement sur le concret pour en saisir la singularité ; le concept, qui vise les déterminations générales de la réalité. L'analyse de l'activité cognitive du sujet a permis l'émergence d'une autre manière de concevoir la connaissance, qui ne s'exprime plus en termes de " représentation » mais d'« intentionnalité ": " le concept est la médiation par laquelle opère l'intentionnalité cognitive lorsqu'elle vise ainsi le réel à travers l'élément de généralité » (Ladrière, 2002). Canguilhem (1966), penseur du vivant et de l'action, réinterroge la connaissance par concept à la lumière de la connaissance (voire d'une philosophie) de la vie. Selon lui, " la vie est sens et concept ", et la connaissance est " une recherche inquiète [...] d'un être insatisfait du sens trouvé » (id., p.223). Le même rapport du concept à la vie, investi philosophiquement, est présent chez Foucault pour qui « former des concepts [...I c'est une façon de vivre dans une 
relative mobilité et non pas une tentative pour immobiliser la vie » $(1985$, p. 13). Le rôle actif et vivant du concept - loin donc de sa fonction contemplative et passive, en tant que représentation mentale abstraite et universelle - est appuyé également par Deleuze \& Guattari (1991) qui associent à leur tour la création de concepts aux " cris philosophiques ".

\section{Objectif de l'étude et problématique}

Qu'un concept soit appréhendé comme une forme rationnelle ou intuitive de connaissance, un outil de réflexion ou une forme de vie, il relève clairement d'une approche philosophique, s'agissant de la conception du concept. En gardant cette perspective conceptualisante, j'ai souhaité observer la présence des concepts dans des pratiques philosophiques conduites avec de jeunes penseurs (appelonsles des " apprentis philosophes ») pour en décrire à la fois la nature et le processus individuel qui préside à leur élaboration.

Je fais ici référence à la pratique de la philosophie avec les enfants, qui se développe depuis maintenant un demi-siècle à partir du programme Philosophy for children (dorénavant P4C). Développé par M. Lipman (1991) et ses collaborateurs aux Etats-Unis, ce programme vise à créer des conditions favorables pour permettre aux enfants de mieux penser en apprenant à philosopher dès leur plus jeune âge. II a été initialement prévu que ce programme soit intégré dans le curriculum scolaire mais, malgré leur essor dans le monde entier ces pratiques philosophiques restent encore en marge des systèmes d'éducation. Autrement dit, l'apprentissage du philosopher n'est que très faiblement reconnu officiellement ; il n'est ainsi que peu associé au devenir de l'élève avant la classe de terminale. ${ }^{4}$

Dans le corpus ${ }^{5}$ de paroles retranscrites, que j'ai choisi pour observer les attitudes pensantes des apprentis philosophes, des élèves âgés de 8 à 14 ans s'exercent au travail conceptuel en dehors de tout cours classique de philosophie. L'approche philosophique dans laquelle s'inscrit cette pratique encourage à choisir une grille de compréhension des concepts qui est axée sur la recherche de sens, que les enfants ou jeunes entreprennent face à une réalité complexe, plutôt que sur l'acquisition de concepts déjà là, définis auparavant et qui seraient destinés à l'élaboration d'un savoir. On appellera ce processus " formation spontanée de concepts ", expression employée à la suite de Bruner (1956/2017) par Barth pour décrire une démarche inventive : « il se peut que le concept ainsi formé n'existe pas selon les conventions, ou qu'il soit 'faux' ; malgré cela il remplit temporairement sa fonction d'organiser le monde pour la personne qui l'a formé » (2013, p.44).

${ }_{4}^{4}$ Pour ce qui est du système scolaire français, la « discussion à visée philosophique » a fait l'objet d'une courte apparition dans les textes de l'éducation morale et civique (2015-2018). Dans certains pays la discipline scolaire " philosophie » n'existe même pas.

${ }^{5}$ II s'agit du corpus recueilli pour la réalisation de ma thèse de doctorat, auprès des élèves de primaire (810 ans) et des élèves de collège (11-14 ans). Pour la description du corpus, v. Fournel (2018, chap. 3 , section 3.3). 
Les démarches en philosophie pour enfants, adaptées à la formation des enseignants ou animateurs, préconisent, grâce à l'exercice du dialogue philosophique, le développement chez l'enfant de la compétence philosophique ${ }^{6}$ de " conceptualiser » (Tozzi, 2007). C'est pourquoi la philosophie apparaît dans ce contexte comme une méthode permettant aux enfants de s'approprier des outils du philosophe afin de mieux conduire leur pensée (Barth, 2003, p. 11). Lipman considérait, pour sa part, différemment le rôle de la philosophie, en voyant en elle un sujet " miscible » à toutes les disciplines, un " guide idéal » dans l'apprentissage de la pensée « du fait qu'elle allie impérativement logique rigoureuse et souplesse "(2006, p. 76).

Si l'activité de conceptualisation est associée sur le plan éducatif à une formation via des outils, on pourrait se demander si les enfants peuvent se vivre dans ce contexte comme des philosophes. Sont-ils capables de produire une pensée conceptuelle spontanée, singulière, sagace et inattendue, lorsqu'ils évoluent dans une réflexion collective ? J'ai l'intuition que cette singularité peut s'exprimer à travers une dimension émotionnelle, qui, mobilisée dans le processus de formation des concepts, est indispensable.

Notre approche cherche à jeter un peu plus de lumière sur l'expression spontanée des pensées subjectives et singulières à l'œuvre dans les pratiques des discussions philosophiques avec les enfants. Si le contexte dialogique et interactionnel de cette praxis invite les chercheurs à s'intéresser particulièrement aux mouvements de pensée collective (Auriac-Slusarczyk \& Colletta (eds.), 2015), il peut sans doute nous permettre aussi d'accéder à une meilleure compréhension de ce qui fait l'articulation entre l'individuel et le collectif, dans l'élaboration d'une pensée philosophique.

\section{Spontanéité du philosopher : émotions et corps en P4C}

On dit que l'enfant est né philosophe mais il ne le demeure pas forcément. Les études montrent que le questionnement n'est plus une activité spontanée à partir de la troisième année de primaire (Dewey, 1916, Lipman \& al., 1980, Daniel, 2008). Les pratiques du dialogue philosophique entre pairs ont été envisagées dans le but de permettre aux enfants de maintenir vif leur questionnement ou de renouer avec celui-ci, d'apprendre à penser en adoptant une posture de philosophe. Pour ce faire, plusieurs habiletés sont à acquérir ou à développer, habiletés qui permettent, selon Lipman (2006), de " muscler » la pensée : définir, distinguer, comparer, justifier, contredire, formuler des hypothèses, faire de raisonnements logiques, etc. Ce sont donc des habiletés cognitives auxquelles s'ajoutent des attitudes et des dispositions (l'étonnement, la curiosité, etc.) ainsi

\footnotetext{
${ }^{6}$ Lipman l'appelle « habileté de pensée » (2006), sans mentionner qu'elle soit spécifiquement philosophique. D'ailleurs la conceptualisation est bien présente dans la démarche scientifique, et parfois même dans celle artistique.
} 
que des habiletés sociales (l'entraide, le dialogue, etc.). En effet, pour Lipman la pensée est moins concernée par l'usage rationnel de nos capacités intellectuelles que par un engagement total dans le processus de recherche et donc dans l'action de la pensée.

De par ses inspirations pragmatistes, la P4C valorise l'expérience de l'enfant, son imagination et la pratique sociale. Elle renvoie ainsi à une vision holistique de la pensée, englobant une pensée critique et rationnelle mais également une pensée créative et attentive, avec une place accordée à l'expression des émotions, voire des sentiments. Le rôle de ces dernières a été mis en évidence en lien avec la formation du jugement (Lipman, 1991 ; Leleux, 2009), le développement psychologique de l'enfant et de la gestion des relations sociales (Giménez-Dasi \& Daniel, 2011). Depuis maintenant plus d'un demi-siècle, de nombreuses études expérimentales ou semi-expérimentales s'attachent à montrer les effets bénéfiques de la philosophie pour enfants sur le développement cognitif, langagier, discursivo-dialogique mais aussi sur la confiance en soi, l'empathie, augmentation de la coopération, un meilleur contrôle des émotions et une meilleure cohésion de groupe. En revanche, très peu d'études s'intéressent à la place des émotions au sein même des pratiques philosophiques depuis les premiers travaux franco-québécois (Schleifer, Daniel, Peyronnet \& Lecomte, 2003 ; Daniel, 2005).

Les émotions sont le plus souvent objets d'apprentissage plutôt qu'objets d'étude pour enquêter sur le rôle des représentations que les enfants se font des émotions afin de mieux les comprendre (Duclos, 2009). Des programmes d'intervention notamment sur la gestion de la colère et de prévention de la violence ont été mis en place mais leur évaluation montre que les enfants apprennent à gérer leurs émotions par la mémorisation d'instructions, sans être en mesure de transférer ces apprentissages à d'autres contextes (Giménez-Dasi \& Daniel, 2011). Cependant, si " éduquer " les émotions veut dire apprendre à les contrôler, comment serait-il possible d'accueillir l'urgence d'une émotion, la spontanéité d'une pensée?

Enfin, dans le champ de la linguistique multimodale, des recherches s'intéressent de plus en plus à l'engagement du corps dans la construction du raisonnement, et donc dans l'élaboration des concepts. Ainsi, les études menées sur la production des gestes co-verbaux dans les interactions montrent que les gestes aident à penser (Colletta, 2004 ; Polo, à paraître) et que la production des métaphores gestuelles, par exemple, joue un rôle important dans l'élaboration des concepts (Lagrange-Lanaspre, à paraître).

En somme, il est possible d'affirmer que les composantes émotionnelle et corporelle ont une place reconnue (bien qu'insuffisamment étudiées) au sein des pratiques philosophiques avec les enfants. Si elles sont habituellement étudiées en lien avec le processus de pensée élaboré dans l'interaction, elles ont été moins explorées en tant que formes subjectives ou singulières d'expression de la pensée. 


\section{Concept et conceptualisation : cadres théoriques}

Parler de formes subjectives d'expression de la pensée, dans le contexte d'une démarche de recherche collective, suppose d'admettre que ces formes peuvent être inachevées et incomplètes. Dans ce type d'interactions étudiées, les enfants ne possèdent pas à l'avance des concepts rigoureux et bien circonscrits, ainsi les conceptualisations peuvent émerger et se construire durant la discussion. Les concepts sont donc envisagés comme des formes en construction qui ont été décrites, dans une recherche récente adoptant une perspective linguistique et pragmatique, comme des « structures formelles verbales liées à la pensée collective en acte » (Fiema, 2014). Cette recherche s'intéressant au raisonnement collectif a mis en évidence la présence de phénomènes de redéfinitions d'un terme de départ qui aboutit à la construction d'un concept, grâce à une mise en commun des significations individuelles. II en résulte des structures formelles de type conceptuel, récurrentes dans le processus d'exploration appelés philosophèmes (Auriac-Slusarczyk \& Fiema, 2013).

Pour se faire une idée de ce que font les enfants quand ils conceptualisent, je présente un extrait qui retranscrit les productions verbales de collégiens et de leur enseignant dans une discussion sur le thème " qu'est-ce que partager ? " (voir l'extrait 1, ci-après). Deux élèves, Maalik et Soliman”, âgés de 11 ans, interviennent dans la discussion lorsque l'enseignant invite les participants à explorer la signification du verbe " partager ».

Extrait 1 : "Qu'est-ce que partager?".

Locuteurs : Malik et Soliman - élèves et l'enseignant

\begin{tabular}{lll}
\hline TP & Locuteur & Parole \\
\hline 84 & Enseignant on s'attaque à ça // ouais qu'est-ce que partager ou qu'est-ce que le \\
\hline$\ldots$ & & \\
\hline 120 & Enseignant ouais // on pourrait déjà s'intéresser à tous les verbes qui se \\
& rapportent à partager \\
\hline 121 & Maalik & $\begin{array}{l}\text { et (il) y a beaucoup de verbes qui ne qualifient pas le mot partager / } \\
\text { moi je voudrais dire que // partager déjà on sait que partager ça veut } \\
\text { dire que c'est pas que pour toi // partager déjà c'est }\end{array}$ \\
\hline 123 & Soliman & $\begin{array}{l}\text { faire profiter les autres } \\
\text { c'est:: / c'est de / c'est:: // en fait on est sûr que c'est pas qu'une } \\
\text { personne qui va / qui va \{un enfant : profiter qui va profiter de / de / } \\
\text { de / de:: // point d'interrogation }\end{array}$ \\
\hline
\end{tabular}

\footnotetext{
7 Les prénoms des participants ont été changés afin de préserver leur anonymat.

${ }^{8}$ Thèmes extraits du corpus (Fournel, 2018), cf. note 4 plus haut.
} 
La proposition de l'enseignant d'explorer les synonymes du verbe (TP 121) sera contestée par Maalik (TP 12) : « (il) y a beaucoup de verbes qui ne qualifient pas le mot partager " (des verbes comme " donner ", " prêter » ont été évoqués dans la discussion plus tôt et on peut supposer que l'élève fait référence à cette recherche antérieure). Maalik propose une autre manière de conceptualiser le partage en identifiant une spécificité de l'action : ce n'est pas qu'une seule personne qui est concerné mais plusieurs : " c'est pas que pour toi " (TP 121). Soliman reformule entre temps ce trait définitoire, sous la forme d'une reprise du discours d'autrui (Roiné, 2016) ainsi : " faire profiter aux autres » (TP 122).

Le concept (conceptus = con-tenir) doit " tenir ensemble » un contenu de pensée, résumant ou unifiant une multiplicité de perceptions, par généralisation et abstraction de traits communs. Il y a des concepts (" rondeur ", " conservation des quantités », etc.) à acquérir chez le jeune enfant ; il y a d'autres concepts (" partager ", " rire ", " identité ", " destin ${ }^{8}$, etc.) qui sont plutôt des concepts à réfléchir. Parmi ces derniers, certains sont communs et ancrés dans le quotidien des participants aux discussions philosophiques (" partage ", " rire ») ; d'autres (" identité ", "liberté ", " destin ") pourraient davantage être associés à des thématiques philosophiques. II reste à préciser ce qui distingue le concept philosophique d'un concept ordinaire ${ }^{8}$. On pourrait également se demander ce qui est véritablement philosophique dans la pratique de la conceptualisation : est-ce le processus de conceptualiser ? Est-ce l'émergence des concepts ou leur élaboration ? Sont-ce les concepts eux-mêmes ?

Il existe un certain nombre de termes équivalents à celui de " concept » (empruntés parfois à la logique, à la linguistique ou à la psychologie) : contenu de pensée, représentation générale, abstraction, universaux, idée générale, substance, signification, signifié, signe, prototype, etc. Pour l'attribut " philosophique ", on pourrait évoquer des propriétés comme : général, problématique, spéculatif, complexe, flou, non-scientifique, non-consensuel, discutable, non-défini, idéologique, etc. Comme je le montrerai par la suite, les cadres théoriques élaborés en P4C s'intéressent davantage au processus de conceptualisation qu'au résultat de cette activité (le concept), et, par conséquent, les formes conceptuelles restent à définir.

Après la présentation des deux principales approches de la conceptualisation en philosophie pour enfants, I'une proposée par Lipman (2003), l'autre par Tozzi (2007), je convoque délibérément un cadre théorique extérieur au paradigme éducatif de la P4C. Si l'analyse linguistique a pu mettre au jour la présence de philosophèmes, de quelle compréhension pourrait se voir enrichie la question de la conceptualisation lorsqu'une conception philosophique du concept est conviée ? L'approche philosophique de Deleuze et Guatari (1991/2005) portant

\footnotetext{
${ }^{8}$ Cette distinction rappelle celle opérée par Vygotski entre concept scientifique et concept quotidien (v. Pensée et Langage, chapitre 5, Étude expérimentale du développement des concepts, pp. 189270).
} 
sur la création des concepts, doit permettre de jeter un regard nouveau sur la spécificité philosophique de la formation des concepts

Je m'appuie donc pour commencer sur les deux cadres conceptuels les plus usités en France. Le modèle de J. Lévine (2014) pourrait être également pris en compte lorsqu'on s'intéresse aux pensées singulières des élèves. Cependant, le modèle centré sur l'expérience du "sujet pensant ", l'enseignant étant hors du cercle de la parole, ne permet pas d'étudier l'interaction qui joue, à mon sens, un rôle essentiel dans l'élaboration de la pensée en opérant un contraste entre des éléments provenant du collectif et des éléments provenant de l'individu.

\subsection{Une réflexion sur les concepts chez Lipman}

Le raisonnement en $\mathrm{P} 4 \mathrm{C}$ est étudié sous l'angle des processus cognitifs à l'œuvre. Ce n'est donc pas l'aboutissement conceptuel qui est recherché lorsque les enfants sont invités à conceptualiser, mais le développement et l'amélioration des possibilités individuelles déjà là. Lipman (2006) les nomme " habiletés de pensée » (thinking skill). Les pratiques éducatives, comme celle de la CRP, sont censées renforcer le processus de pensée dans quatre grandes aires de compétences réflexives : 1 . raisonnement, 2 . recherche, 3 . organisation de l'information et 4. traduction. Rangée du côté de l'organisation de l'information, la conceptualisation porte d'une part sur les choses, i.e. " grouper des choses en fonction de leur similarité ", les concepts étant alors ainsi considérés comme des entités qui permettent de manier la pensée : « La conceptualisation concerne l'organisation de l'information dans un réseau de relations à analyser et à clarifier par la suite, de manière à pouvoir les [entités] utiliser en vue d'une meilleure compréhension et d'un meilleur jugement. » (2006, p. 178). D'autre part, la conceptualisation porte sur les concepts eux-mêmes et leur mise en relation : " la pensée conceptuelle met en relation des concepts les uns avec les autres, pour arriver à des principes, des critères, des arguments, des explications, etc. " (ibid.).

Inspiré par l'analyse du langage ordinaire d'Austin (1979), vue comme une enquête philosophique sur les usages des mots, Lipman propose de conceptualiser avec les enfants à l'aide d'un outil pédagogique / didactique qui est la " cible " 9 . (voir la Figure 1, ci-après). Cet outil permet de travailler sur la signification des concepts pour dégager des unités de sens à partir du matériau linguistique, de la façon suivante : au milieu de la cible on place l'aire du concept visé (ex : « partager ») et dans l'air concentrique extérieur on place son antonyme (« ne pas partager »). Les deux aires sont séparées par une " aire floue " qui accueille des états intermédiaires (quelque chose qui n'est pas tout à fait partagé, par exemple " prêter »). Les synonymes placés dans la zone centrale (" ne pas garder que pour soi

\footnotetext{
${ }_{9}$ Un modèle de cible est proposé par M. Lipman et A. M. Sharp dans le Guide d'accompagnement du roman Kio et Augustin. S'étonner devant le monde (Wondering at the world: instructional manual to accompagny Kio \& Gus. Lanham), Chap. 1, épisode 4 : "Le développement des concepts ».
} 
", " faire profiter aux autres », etc.) sont censés renforcer la signification générale du concept " partager ${ }^{10}$ Le travail de recherche sur les concepts consiste à vérifier dans quelles circonstances et avec quelles intentions nous utiliserions chacune des expressions inventoriées.

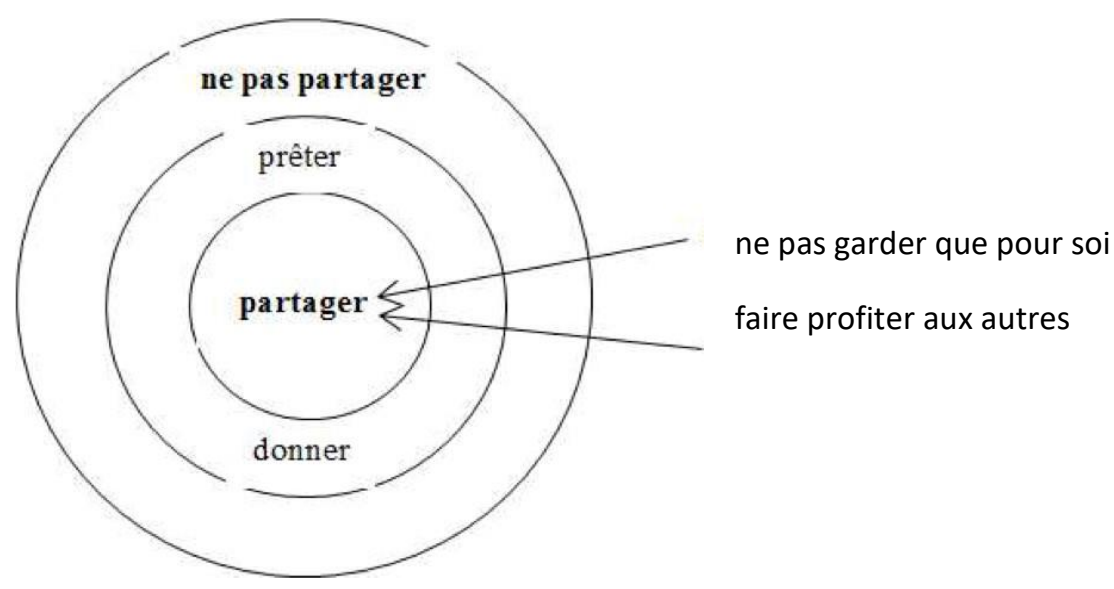

Figure 1 : Analyse du concept " partager » inspirée de Lipman, 1995, p.65, reprise de Lipman \& Sharp, Wondering at the World, Lanham, Md., University of Press of America, 1986, pp. 74-75.

Lorsque la réflexion sur les concepts s'accompagne d'une mise en discussion des "critères et normes qui entourent des concepts", permettant de distinguer les processus de pensée corrects des processus de pensée incorrects (2006, p. 161), le travail de conceptualisation se confond avec l'activité philosophique. Le rôle de la philosophie, d'après Lipman (1991), est de fournir des schémas de référence - logique, métaphysique, éthique, esthétique, épistémologie, etc. - pour penser la complexité, et pour penser en complexité.

Pour revenir à l'extrait " partager » (cf. Extrait 1, plus haut) et exploiter le langage lipmanien, on pourrait dire que Maalik remet en question l'approche par synonymie proposée par l'enseignant et propose une approche par critères (aspect épistémologique) en prêtant attention à ce que le partage suppose comme implication pratique (dimension éthique) : " ne pas garder pour soi », ou « faire partager aux autres $»$.

\footnotetext{
${ }^{10}$ Ce type de schéma est illustré en 1986 et 1995 sur le concept « mouillé " versus " pas mouillé »
} de manière très éclairante. 


\subsection{La compétence philosophique de conceptualisation chez Tozzi}

Tozzi (2007) considère la conceptualisation comme une compétence spécifiquement philosophique, articulée à celle de la problématisation et l'argumentation. II la définit comme " une construction conceptuelle à teneur philosophique " à partir des représentations individuelles spontanées exprimées. L'auteur met en balance d'une part les limites que rencontre l'enfant lorsqu'il s'exerce à la conceptualisation - expérience limitée, tendance à ne penser qu'à partir de sa propre expérience, exemples connus, généralisation hâtive, manque de vocabulaire précis et de structures syntaxiques et rationnelles; d'autre part, les potentialités qui permettent au sujet d'apprendre à conceptualiser. L'étayage de l'adulte est présenté comme indispensable pour favoriser le passage de l'exemple personnel à la définition, celui du vécu à la notion, celui du concret à l'abstrait, en faisant toutefois mention d'une vigilance à repérer aussi l'effet de l'étayage mutuel, effectif et exercé par les pairs, qui joue également son rôle.

Au fond, tout comme chez Lipman, la conceptualisation passe par un travail sur les mots et les distinctions, pour rapprocher le semblable et discriminer le différent (ex. donner/prêter, etc.), et par l'exercice de la définition. La définition " verbo-conceptuelle » d'une notion permettant de généraliser et d'abstraire du réel des attributs spécifiques, fait écho à l'approche prédicative de type aristotélicienne. Tozzi (1993) met en perspective d'autres approches en conceptualisation et fournit des éléments complémentaires d'analyse de l'activité de conceptualiser en P4C : a) "l'approche par signification », lorsque la conceptualisation se fait par l'explication du sens langagier (définition de dictionnaire) ; b) " l'approche par unité problématique de sens ", lorsqu'on explore l'intelligibilité d'une notion à travers la polysémie de ses champs d'application; c) "l'approche métaphorique ", qui fait appel au sens implicite via des images, symboles, allégories, mythes, etc. qui évoquent la notion ; enfin d) " l'approche critique de l'opinion " consistant à exprimer et mettre en question les représentations spontanées, par confrontation avec les pairs ou les grands auteurs.

Lorsqu'on relit l'extrait " partager » (cf. Extrait 1, plus haut) à la lumière de cette interprétation, on peut mettre à nouveau en évidence l'initiative conceptualisante qu'engage l'élève Maalik. Alors que l'enseignant proposait une modalité de conceptualiser " par signification » (cf. a), plus haut), l'élève choisit de travailler la notion en compréhension (vs. extension) et d'adopter une approche de type plutôt aristotélicien, amenant à définir ce qui caractérise plus spécifiquement le " partage ", i.e. pour cet élève, "faire profiter aux autres".

Les deux cadres théoriques, proposés par Lipman puis Tozzi, permettent de décrire, selon moi, a posteriori le processus de conceptualisation mais fournissent peu ou pas d'éléments pour envisager le concept dans sa valeur singulière et subjective. II m'a paru donc utile d'intégrer à cette analyse théorique un troisième cadre de référence qui convoque clairement la « vie " à la table de la cognition. 


\subsection{Le concept comme « cri philosophique »chez Deleuze}

Le registre théorique d'inspiration phénoménologique, que les sciences cognitives ont repris à travers la notion d'énaction (Varela et al., 1991), s'articule autour des idées suivantes : les connaissances et le savoir n'émergent que sur le fond d'un arrière-plan de compréhension ; cette compréhension est enracinée dans notre relation à l'environnement, inséparable de notre corps, de notre langage et notre culture. C'est ce qui permet à l'individu de donner du sens au monde dans lequel il évolue, en tant que présence praxique, pas seulement contemplative ou représentative. C'est d'autant plus valable chez l'enfant, pour qui l'activité cognitive est également sensori-motrice et émotionnelle. Dans les multiples choix théoriques possibles reliant le concept au vivant, je choisis donc la tradition ouverte par Canguillem (1966), replaçant le rapport de la connaissance à la vie, du concept à la réalité. À sa suite, des auteurs comme Deleuze (1980) et Foucault (1985) concevront, eux-aussi, le concept comme une forme de vie. C'est la proposition deleuzienne du concept non-discursif, identifié au cri, qui est ainsi retenue pour mon analyse.

Pour définir le concept, Deleuze (1984) propose une comparaison qui semble éclairante pour la compréhension des formes singulières d'expression(s) de la pensée. Il le fait dans un séminaire sur Leibniz, donné le 15 avril 1980, à I'Université Paris 8 :

[...] d'une certaine manière je me dis que les concepts sont des choses tellement vivantes, c'est vraiment des trucs qui ont quatre pattes, ça bouge, quoi. C'est comme une couleur, c'est comme un son. Les concepts, c'est tellement vivant que ce n'est pas sans rapport avec ce qui pourtant paraît le plus loin du concept, à savoir le cri (Vincennes, 1980) ${ }^{11}$.

Le concept est au discours ce que le cri est au chant — voilà donc une approche métaphorique de conceptualisation du concept. En associant les formes de pensées à des objets de perceptions (sons, couleurs, cris), elles sont des empreintes de corporéité et de subjectivité, et l'auteur propose de cette manière une vision incarnée du concept (cf. Varela et al., 1991).

Deleuze va plus loin encore dans la comparaison entre le concept et le cri. Cette fois-ci, c'est dans un autre cours, consacré au thème Cinéma et Pensée (1984) où il propose une analogie entre l'exploration des concepts par le philosophe et la démarche de l'ornithologue qui étudie les productions vocales chez les oiseaux :

Chacun sait que chez les oiseaux, on distingue les cris et les chants. Le cri d'alarme, par exemple, n'est pas un chant, comment là les ornithologues auraient beaucoup à nous apprendre s'ils arrivaient à nous donner de fermes distinctions entre le chant et le cri. Mais je peux dire que de même dans la

\footnotetext{
${ }^{11}$ Cours sur Leibniz / Vincennes - St. Denis / 15 avril 1980, dont l'exposé a été enregistré et retranscrit. Consulté sur https://www.webdeleuze.com/textes/48.
} 
philosophie il y a des discours et que les discours ne sont pas la même chose que les cris, les discours c'est le chant des philosophes (1984) ${ }^{12}$.

Sous l'effet des émotions, les cris peuvent être de joie, de colère, de rage, d'effroi, d'étonnement, de surprise, d'admiration, d'angoisse, de terreur, d'horreur, etc. Chez l'humain le cri traduit également l'expression d'un état moral ressenti intensément, comme c'est le cas d'un cri de révolte, pour exemple. Produits pour prévenir autrui, ou pour exprimer un avis qui conteste ou s'oppose, certains cris peuvent aussi rester à l'intérieur ${ }^{13}$. Le cri philosophique deleuzien serait donc une interpellation, une réalité philosophique vivante, communiquée ou non, mise en discours ou non. C'est le sens du rapport de la comparaison introduite dans le second extrait de Deleuze : tout comme le cri se distingue d'un chant ou d'une composition musicale, le cri philosophique se distingue par la singularité de son acte d'un mode de pensée discursif qui, lui, organise et construit des suites d'énoncés. Au demeurant, pour Deleuze, le concept n'est pas la pensée mais « un système de singularités prélevé sur un flux de pensée » (Deleuze, Vincennes, 1980).

Cette distinction entre cri et discours sera intégrée en une vision philosophique du concept exposée par Deleuze et Guattari dans Qu'est-ce que la philosophie ? (1991/2005). On peut identifier dans leur ouvrage trois principes qui sous-tendent la vision du concept comme forme de vie : (a) la philosophie est une activité précise, singulière, de création de concepts (p.12) ; (b) les concepts ont besoin de personnages conceptuels (p.8) ; (c) la philosophie a horreur de discussions (p.34). Or cette vision n'est pas celle avec laquelle nous avons l'habitude de réfléchir au sujet des pratiques philosophiques avec des enfants, dont les principes sont, à bien y regarder, quasiment opposés : (a) la pratique philosophiques (avec différents publics, dont enfants, jeunes) est une activité collective de construction de concepts ; (b) l'auteur d'un concept ou d'une pensée construite collectivement n'est pas toujours identifiable ; (c) la pratique philosophique se nourrit de la discussion.

Deleuze (1980) propose d'identifier conceptuellement les cris à travers deux principales formes : les " cris de la raison » et les "cris de la déraison ». Les premiers prennent la forme d'une évidence. Chez Aristote, il ne fait pas doute qu'il existe une cause première, autrement il faudrait remonter à l'infini d'une cause vers une autre, dans une régression à l'infini. Le cri déjà chez Aristote est une interpellation : " il faut bien s'arrêter quelque part " et admettre des concepts ultimes. Il en est de même de l'interpellation chez Leibniz qui s'impose comme une évidence car il n'est pas pensable selon lui qu'il puisse y avoir quelque chose qui n'a pas de raison. Les « cris de la raison " sont donc des évidences ; ils ne sont pas des arguments, car il ne s'agit pas de convaincre. Ils ne sont pas non plus des opinions, car on ne peut pas demander des raisons pour les justifier. On pourrait les

\footnotetext{
${ }^{12}$ Cinéma et pensée, cours 67 du 30/10/1984 -2, consulté le 21 septembre 2018 à http://www2.univparis8.fr/deleuze/article.php3?id_article $=5$

${ }_{13}$ Trésor de la Langue Française informatisé (TLFI), art. « cri ».
} 
rapprocher du sentiment ou de l'intuition, et en tout cas, d'une réponse qui exclut toute problématisation.

Le second type de cris - «cris de la déraison » - sont des formes de lutte contre toute évidence. Deleuze (1980) cite le cri lancé par Dostoïevski : « Je veux, je veux que on me rende compte de chaque victime de l'Histoire, je ne serai pas tranquille et je ne vous laisserai pas tranquille tant que vous n'aurez pas rendu compte de chaque victime de l'histoire "; ou celui de Chestov, qui demande avec le psaume "Comment, jeune, garder pur son chemin ? ". Chez ces penseurs, la colère et la révolte ont pour fonction de faire penser. On pourrait associer ces cris au pressentiment, qui échappe lui aussi à toute contestation rationnelle.

\section{Analyses des formes singulières de conceptualisation dans le corpus}

A partir de ce triple cadre conceptuel, je me propose de vérifier dans le corpus étudié (voir ci-après) si des formes singulières de conceptualisations apparentées aux cris philosophiques peuvent être identifiées chez les apprentis philosophes. Est-ce que les enfants/jeunes ont quelque chose à crier, et si oui sous quelle forme?

Dans le corpus de discussions philosophiques à notre disposition, j'ai sélectionné deux séances réalisées et enregistrées en contexte scolaire, une en secondaire et l'autre en primaire. La première est la séance déjà citée, "Qu'est-ce que partager ? ", enregistrée au collège avec des élèves de $6^{\mathrm{e}}$ (11-12 ans), dans laquelle les élèves explorent ensemble le sens du terme " partager ". La seconde, enregistrée en primaire avec des élèves de CE2-CM1 (8-10 ans) a utilisé comme support de réflexion la lecture le premier chapitre du roman philosophique « Pixie ", écrit par Lipman (1989). Dans ce dernier cas, l'enseignante a invité les élèves à exprimer ce qui les a étonnés, ou ce qu'ils aimeraient comprendre au sujet de I'histoire. Dans le protocole d'une CRP, on appelle cette étape une phase de questionnement (Fournel, 2018).

\subsection{Formes conceptuelles du « partager}

Dans un premier temps, je vais m'intéresser à l'activité de conceptualisation ${ }^{14}$ en appui sur deux extraits de la discussion sur le partage, tenue au collège. Le premier échange (qui a déjà fait l'objet de nos observations, en marge de l'extrait 1) fait ressortir deux types d'approche de la conceptualisation : 1. une proposée par l'enseignant - approche par la signification - et 2 . une autre proposée par les élèves - approche prédicative ou par identification de critère. Un

\footnotetext{
14 V. notre section 4.2. (Tozzi, 1993) : description des différentes approches de la conceptualisation.
} 
deuxième échange permet d'accéder de manière complémentaire au processus de conceptualisation à l'œuvre, et de mettre l'accent, cette fois-ci, sur les réalisations individuelles des deux élèves impliqués dans la discussion (Maalik et Soliman); c'est le passage où Maalik dit : " partager déjà on sait que partager ça veut dire que c'est pas que pour toi » (voir ci-dessous l'extrait 1, dans Tableau 1).

Tableau 1. Analyse conceptualisation. Extrait I : Q Qu'est-ce que partager ? Locuteurs : Malik et Soliman - élèves et l'enseignant

\begin{tabular}{lllc}
\hline TP & Locuteur & Parole & $\begin{array}{c}\text { Analyse } \\
\text { conceptualisation }\end{array}$ \\
\hline 120 & Enseignant & $\begin{array}{c}\text { ouais // on pourrait déjà s'intéresser à tous les } \\
\text { verbes qui se rapportent à partager }\end{array}$ & $\begin{array}{c}\text { par exploration } \\
\text { sémantique } \\
\text { lexicale }\end{array}$ \\
\hline 121 & Maalik & $\begin{array}{l}\text { et (il) y a beaucoup de verbes qui ne qualifient } \\
\text { pas le mot partager / moi je voudrais dire que } \\
\text { partager déjà on sait que partager ça veut dire }\end{array}$ & $\begin{array}{c}\text { prédicative } \\
\text { interpellation }\end{array}$ \\
\hline 122 & Soliman & que c'est pas que pour toi // partager déjà c'est & \\
\hline 123 & Maalik & $\begin{array}{l}\text { c'est:: / c'est de / c'est:: // en fait on est sûr que } \\
\text { c'est pas qu'une personne qui va / qui va }\{\text { un } \\
\text { enfant : profiter }\} \text { qui va profiter de / de / de / } \\
\text { de:: // point d'interrogation }\end{array}$ & prédicative \\
\hline
\end{tabular}

Légende : est souligné en gras l'énoncé central à l'origine de

l'analyse interprétative proposée

Rappelons brièvement ce qui a été observé précédemment (section 4, analyse de l'extrait 1) : alors que l'enseignant propose aux élèves (en TP 120) une démarche de conceptualisation de type exploration sémantico-lexicale (par synonymie), l'élève Maalik rejette cette proposition et procède autrement. II identifie un "prédicat " $(\mathrm{P})$ (dans le sens aristotélicien du terme, sur un plan à la fois linguistique et logique) qu'il attribue au " sujet » (S) partager. Dire $S$ est $P$ revient à dire : " partager est pas que pour toi ». Soliman reformule par " c'est faire profiter aux autres ", ce que, dans la logique de Frege (1879-1925/1994), on appellerait une "fonction propositionnelle».

Dans le processus de cette élaboration conceptualisante, la posture énonciative de Maalik (en TP 121) attire attention. Sur le plan énonciatif, la proposition de l'élève est une mise en relief de l'assertion du dire par une forme du vouloir : " moi je voudrais dire que " (sont soulignés les termes sur lesquels je fais porter l'analyse et l'interprétation). C'est un dire que le locuteur expose soigneusement, pour en souligner la certitude - « on sait » : " partager ça veut dire que c'est pas que pour toi » (idem). Je rapprocherais cette assertion d'une évidence, 
à l'image d'un concept figé qu'il s'agit de rappeler. Ce concept est dépourvu d'authenticité, et n'est ni une opinion, ni un argument. Il s'apparente à une interpellation de la raison : vous ne pouvez pas nier que partager ça veut dire que c'est pas que pour toi. Toutefois, pourquoi s'agirait-il d'un concept et non pas juste d'une doxa?

Je propose de poursuivre le fil de la discussion à laquelle participent les mêmes interlocuteurs, et en particulier se focaliser sur l'échange ci-dessous (Extrait 2 , Tableau 2). Après cette proposition de conceptualisation prédicative formulée conjointement par les deux élèves Maalik et Soliman, une vingtaine de tours de parole plus loin l'enseignant revient sur ce moment de réflexion.

Tableau 2. Analyse conceptualisation. Extrait 2 : "Qu'est-ce que partager?". Locuteurs : Soliman - élève et l'enseignant.

\begin{tabular}{|c|c|c|c|}
\hline$T P$ & Locuteur & Parole & $\begin{array}{c}\text { Analyse } \\
\text { conceptualisation }\end{array}$ \\
\hline 142 & Enseignant & $\begin{array}{l}\text { alors (il) y a un truc // (il) y avait } \\
\text { profiter mais // toi tu avais quelque } \\
\text { chose à dire sur le mot profiter // j'ai } \\
\text { / j'ai entendu ta réflexion et je la } \\
\text { trouve intéressante donc vas-y }\end{array}$ & $\begin{array}{l}\text { par exploration } \\
\text { sémantique lexicale }\end{array}$ \\
\hline 143 & Soliman & $\begin{array}{l}\text { euh en fait c'est plutôt faire profiter } \\
\text { les autres mais je voulais euh : } \\
\text { casserce que j'ai dit parce que tu } \\
\text { peux / tu peux partager aussi des } \\
\text { dettes donc c'est aussi des fois pas } \\
\text { sympa pour les autres donc ça / } \\
\text { c'est pas toujours positif le partage }\end{array}$ & $\begin{array}{l}\text { autocritique } \\
\text { par unité } \\
\text { problématique de } \\
\text { sens }\end{array}$ \\
\hline 144 & Enseignant & $\begin{array}{l}\text { et pis c'est pas / et pis tu dis que } \\
\text { finalement profiter / estce que tu } \\
\text { profites des dettes // c'est ça que tu } \\
\text { voulais dire? }\end{array}$ & \\
\hline 145 & Soliman & $\begin{array}{l}\text { non non // donc ça ne qualifie pas } \\
\text { complètement le mot partager }\end{array}$ & prédicative \\
\hline
\end{tabular}

L'enseignant a été visiblement interpelé par la proposition de type reformulation de Soliman et intervient dans le déroulement des échanges pour encourager l'élève à aller plus loin dans sa réflexion (TP142) : " tu avais quelque chose à dire sur le mot profiter [...I vas-y $"{ }^{15}$ L'enseignant donne l'impression de rester dans une démarche d'exploration sémantique lexicale, en s'intéressant au nouveau verbe proposé : « profiter ». La suite est surprenante, car l'élève convoque

\footnotetext{
${ }^{15}$ A noter que la formule « vas-y » introduit un mouvement de l'enseignant qui, inconsciemment sans doute, enjoint l'élève à visiter " son monde ».
} 
encore une nouvelle approche de la conceptualisation (T143). Soliman remet en question le critère qu'il avait proposé précédemment (" faire profiter aux autres ", en TPI 22) et procède, dans les termes de la classification de Tozzi (v. section 4.2), à une approche " critique " de la conceptualisation. Pour rappel, celle démarche consiste à exprimer et mettre en question sa représentation spontanée, par confrontation avec les pairs. En TP 143, Soliman opère une telle critique, par rapport à son propre raisonnement, assortie d'un argument : « je voulais casser ce que j'ai dit parce que tu peux/ tu peux partager aussi des dettes ". II s'agit donc d'une " autocritique " qui aboutit à une conceptualisation différente : le concept « partager " n'est pas toujours positif. C'est une manière d'aller à l'encontre des évidences, et même de ses propres évidences plus précisément, ce qui témoigne d'une capacité à s'interpeller soi-même autant que les autres.

Ce deuxième extrait offre également la possibilité de voir à l'œuvre d'autres manières de conceptualiser, comme l'exploration des champs d'application du " partager ", par exemple dans le cas des dettes le partage n'est pas souhaitable (TP 123) ; ou encore un retour à l'approche prédicative, lorsque Soliman conclut que " faire profiter aux autres » ne qualifie finalement pas le mot " partager » (TP 145). La recherche de conceptualisation peut continuer.

On voit à travers ces deux extraits que les élèves sont capables d'explorer plusieurs manières de conceptualiser, alors que l'enseignant semble cantonné à l'idée qu'il s'est déjà faite de la démarche à prendre. Non seulement les élèves ne restent pas dans le cadre imposé mais ils sont capables d'interpeller et de s'interpeller, de proposer leurs intuitions. On ne peut cependant pas attester que ces apprentis-philosophes soient créateurs de concepts, à la manière de Deleuze.

\subsection{Formes conceptuelles $d u$ «bras endormi »}

Dans la discussion du corpus primaire, un passage met en scène l'une des élèves, Alice, qui se questionne à la suite de la lecture du chapitre 1 de Pixie. C'est d'ailleurs l'enseignante qui invite les élèves à identifier et exprimer ce qui les a intéressés ou intrigués dans cette histoire: " ... alors // vous vous souvenez la semaine dernière je vous avais posé des questions et je vous avais demandé de réfléchir un $p(e) t i t$ peu // à // ce que vous avez trouvé bien intéressant dans cette histoire // qu'est-ce qui vous a plu // dans l'histoire de Pixie... " (TP 12). Non seulement Alice a été interpellée et intéressée par le passage du bras endormi de Pixie (" Avez-vous déjà eu le bras endormi ? N'est-ce pas étrange ? " ${ }^{16}$ ), mais elle

\footnotetext{
${ }^{16}$ Lipman, Pixie, chap.I, p.9. Voici le passage entier : « ...j'avais le menton dans la main et le coude sur le pupitre. Je ne sais pas combien de temps je suis restée comme cela, mais c'était certainement longtemps. Tout à coup, je me suis rappelé que j'étais en classe. Et alors je me suis rendu compte de quelque chose de bizarre. Savez-vous ce que c'était ? Mon bras était engourdi. C'est comme s'il était endormi. Même aujourd'hui je ne comprends toujours pas. Si tout moi était éveillé, comment une partie de moi pouvait-elle dormir ? Et c'est vrai que le bras était endormi. Je ne pouvais pas m'en servir. C'était comme s'il était simplement accroché à mon épaule. Je ne le sentais même pas, sauf peut-être un léger picotement. Avez-vous déjà eu le bras endormi ? N'est-ce pas étrange ? C'est comme s'il ne vous appartenait même pas! Comment une partie de vous-mêmes peut-elle ne pas
} 
interpelle à son tour, durant la discussion ses camarades et l'enseignante à ce sujet (v. T27 et T31, dans Tableau 3, Extrait 3 ci-après).

Tableau 3. Extrait 3 : " Pixie » (CE2-CM1).

Locuteurs : Alice - élève et l'enseignante

\begin{tabular}{llll}
\hline TP & Locuteur & Parole & \multicolumn{1}{c}{$\begin{array}{c}\text { Analyse } \\
\text { conceptualisation }\end{array}$} \\
\hline 27 & Alice & $\begin{array}{l}\text { j'aimerais bien savoir // comment il bah son bras il par analogie } \\
\text { peut être endormi et comment une personne qui } \\
\text { s'est endormie ne puisse pas le bouger alors } \\
\text { qu'elle peut bouger sauf son bras }\end{array}$ \\
\hline 28 & Enseignante & alors // tu peux répéter s'il te plait \\
\hline 29 & Alice & bah euh elle dit qu'il était endormi \\
& & qu'elle ne pouvait plus le bouger \\
\hline 30 & Enseignante & hm \\
\hline 31 & Alice & bah: c'est intéressant et j'aimerais bien savoir \\
& & $\begin{array}{l}\text { euh et comprendre euh bah euh comment } \\
\text { s'est arrivé euh ça }\end{array}$ \\
\hline
\end{tabular}

Alice aimerait comprendre comment il est possible qu'un bras soit endormi, dans le sens de ne plus pouvoir le bouger (TP 27). On pourrait s'attendre à ce que la fillette cherche une explication au phénomène d'engourdissement ou d'anesthésie du bras. D'ailleurs, dans la suite des échanges, plusieurs camarades et l'enseignante elle-même vont tenter de donner une explication scientifique au phénomène, en évoquant un problème de circulation du sang ( $v$. Fournel, à paraitre). Cependant, tout comme chez le personnage Pixie, il me semble que c'est davantage le rapport partie/tout, chez un être vivant qui bouge, qui est ici interrogé ${ }^{17}$. Si notre intuition est bonne, alors la démarche d'Alice peut être caractérisée comme une tentative de conceptualiser le rapport partie/tout en passant par l'image du rapport entre corps et esprit. J'ai appelé cette modalité dans mon analyse (Tableau 3) : approche de conceptualisation par analogie. On peut, me semble-t-il, reconstituer le raisonnement d'Alice comme suit : si on bouge son corps entier alors on bouge aussi chaque partie du corps (dont le bras) ; or une des parties (le bras) ne bouge plus. Cette dernière information vient perturber sa croyance

vous appartenir ? Vous vous appartenez tout entier! Mais voyez-vous, c'est cela qui m'intrigue. Ou bien mon corps et moi sommes identiques, ou bien nous ne le sommes pas. Si mon corps et moi nous sommes identiques, alors il ne peut m'appartenir. Et si mon corps et moi nous sommes différents, alors qui suis-je ? ".

${ }_{17}$ Le problème soulevé par Alice est posé explicitement par le personnage au sein de l'histoire de Pixie. 
selon laquelle le corps fonctionne comme un engrenage qui met tout en mouvement. D'où son étonnement. D'où son interpellation (soulignée en gras dans le tableau 3).

Alice gardera vive son questionnement et son interpellation, qu'elle reprendra dans la suite de la discussion pour le reformuler à plusieurs reprises, sans vraiment y arriver (voir l'extrait 4, ci-après) :

Extrait 4 : « Pixie » (CE2-CM1).

Locuteurs : Alice - élève et l'enseignante

\begin{tabular}{|l|l|l|}
\hline TP & Locuteur & Parole \\
\hline 51 & Alice & $\begin{array}{l}\text { comment un /euh un bras peut être endormi et qu'on peut } \\
\text { bouger le corps }\end{array}$ \\
\hline 52 & Enseignante & $\begin{array}{l}\text { alors comment on peut continuer à bouger son corps tout } \\
\text { en ayant }\end{array}$ \\
\hline 53 & Alice & un bras où peut pas bouger (en)fin bah une (xx) \\
\hline 54 & Enseignante & $\begin{array}{l}\text { une partie de son corps // donc comment c'est possible // } \\
\text { de bouger son corps }\end{array}$ \\
\hline 55 & Alice & $\begin{array}{l}\text { non // en fait c'est euh // comment elle peut avoir le bras } \\
\text { endormi }\end{array}$ \\
\hline 56 & Enseignante & alors elle c'est Pixie \\
\hline 57 & Alice & $\begin{array}{l}\text { comment elle peut plus bouger / Pixie comment elle peut } \\
\text { plus bouger son bras }\end{array}$ \\
\hline 58 & Enseignante & alors ce serait plutôt pourquoi \\
\hline 59 & Alice & oui pourquoi Pixie ne peut plus bouger son bras \\
\hline
\end{tabular}

Dans l'interprétation que je propose, Alice essaye de conceptualiser le rapport entre le corps et l'esprit (incluant l'expérience subjective et la conscience de l'existence du corps), sans parvenir à une formulation claire de ce qu'elle ressent (peut-être aussi dans son for intérieur) comme un inconfort épistémique. On ne pourrait associer sa formulation ni au cri de l'évidence, ni à celui de la révolte, mais peut-être à un cri d'étonnement dont seule l'urgence et l'émotion s'exprimeraient pour le moment.

Si l'on admet les prémices d'un travail individuel de conceptualisation chez cette jeune locutrice, alors on pourrait dire que dans les passages analysés il ne s'agit pas de création de concepts, dans le sens où Deleuze et Guattari évoquaient le terme l'associant au " cri philosophique ", mais plutôt de l'émergence d'un concept incarné. Dans le contexte éducatif des discussions philosophiques, le rôle de l'animateur serait alors d'accompagner à la fois l'éclosion de ces formes d'expression spontanée dans leur singularité et l'élaboration collective de conceptualisations partagées, plus aptes pour patiemment s'engager vers un approfondissement ou vers la définition. L'urgence face à la patience, on pourrait dire. Ne sont-ce pas là deux traits qui conviennent au philosophe? 


\section{Conclusion et discussion}

Dans le cadre des analyses proposées dans cette étude, qui restent limitées en nombre et fortement interprétatives, j'ai pu remarquer que la pratique de la discussion philosophique donne l'occasion à certains élèves d'exprimer des formes de pensée singulières sur le fond d'un processus de pensée collectif. Ainsi, j'ai pu identifier des formes d'expression - des concepts incarnés et des questions - qui s'apparentent à des interpellations vivantes faisant écho au vécu des locuteurs (incluant leur expérience, leurs croyance et émotions). II s'agit de démarches d'accès au concept qui s'expriment avec l'urgence d'une évidence (" on sait que partager ça veut dire que c'est pas que pour toi »), d'une contestation (" c'est pas toujours positif le partage »), ou d'un engagement personnel dans le savoir (" j'aimerais comprendre »). « Comment est-ce possible que le bras soit endormi alors que le corps entier peut bouger ? " : c'est finalement une interrogation vivante de la pensée qui prend comme conscience de sa dimension incarnée, de son lien à la vie. Cela conduit à la définition que Foucault avait donné de l'activité philosophique : « former des concepts I...] c'est une façon de vivre dans une relative mobilité et non pas une tentative pour immobiliser la vie $"$.

Bien que ces " cris " ou interpellations enfantines ne soient pas des créations de concepts, dans le sens de Deleuze et Guattari - comme portant la signature des auteurs philosophes - ils méritent néanmoins, selon moi, d'être entendus et encouragés par les éducateurs. Les enfants ont été longtemps considérés dans l'histoire de la philosophie comme des êtres " mineurs ", incapables de recevoir les lumières de la raison. Le programme éducatif $\mathrm{P} 4 \mathrm{C}$ remet fortement en cause cette limitation de principe. Nonobstant, il ne s'agit pas de voir des concepts philosophiques dans toute interpellation venue de la part de l'enfant, ni de surévaluer les capacités de l'enfant à former des concepts nouveaux.

Réfléchir sur les concepts et les discuter ne vaut pas création individuelle. Cependant cela pose la question, au sein même de la philosophie pour enfants, de la place de la pensée individuelle et rétroactive dans une démarche qui privilégie la construction collaborative orale de la pensée et des concepts, dans et via les interactions. On peut imaginer que l'introduction d'un passage à l'écriture philosophique peut contribuer à renforcer l'originalité des pensées en développement, ce, par une incitation à l'expression d'une pensée singulière ${ }^{18}$.

Aussi, la vision de Deleuze et Guatari sur l'incompatibilité entre l'activité de création de concepts philosophiques et la discussion mérite d'être examinée, car elle peut jeter un regard nouveau sur le rapport entre pensées individuelles et pensée collective telles que tissées en philosophie pour enfants. De plus, s'interroger sur la posture de l'adulte face aux interpellations " vivantes " des apprentis philosophes, au sein de ces pratiques, permettra, je pense, d'examiner

\footnotetext{
${ }^{18} \mathrm{~A}$ ce sujet, des études existent déjà ou sont en cours, voir les travaux de Hélène Maire et Emmanuèle Auriac -Slusarczyk et al., conduits au sein de I'Université Clermont Auvergne.
} 
plus loin les conditions de possibilité d'une approche positive des " cris philosophiques » en P4C.

\section{BIBLIOGRAPHIE}

Auriac-Slusarczyk, E. \& Maire, H. (à paraitre). Sur les pas de Lipman, philosopher à l'école. Une histoire scientifique à connaitre. In Philosopher avec les enfants : fabrique de l'apprendre, fabrique du savoir, Clermont-Ferrand : Presses Universitaires Blaise Pascal.

Auriac-Peyronnet, E., \& Coletta, J.-M. (Éd.). (2015). Les ateliers de philosophie : une pensée collective en acte. Clermont-Ferrand, France : Presses universitaires Blaise Pascal, Collection Sphère Educative.

Auriac-Slusarczyk, E., \& Fiema G., (2013). Raisonner et discuter : définitions et principe d'étude pragmatique du corpus philosophèmes, Cahier du LRL, 5, 41-74.

Austin, J.-L. (1979). Philosophical Papers [1961], éds. J. O. Urmson \& G. J. Warnock, London : Oxford University Press, $3^{\mathrm{e}}$ édition.

Barth, B.-M. (2013). L'apprentissage de l'abstraction. Montréal; Paris: Retz.

Bruner, J. S., Goodnow, J. J., \& Austin, G. A. (1956/2017). A Study of Thinking. New York: Routledge.

Canguilhem, M. G. (1966). Le concept et la vie. In : Revue Philosophique de Louvain. $3^{\mathrm{e}}$ série, tome 64, $\mathrm{n}^{0} 82$, 1966. pp.193-223.

Colletta, J.-M. (2004). Le développement de la parole chez l'enfant âgé de 6 à 11 ans: corps, langage et cognition. Hayen : Editions Mardaga.

Damasio, A. R. (2010). L'erreur de Descartes. La raison des émotions. Paris : Odile Jacob.

Daniel, M.-F. (2008). Learning to philosophize: Positive Impacts and Conditions for Implementations. In: Thinking: The Journal of Philosophyfor children. Vol. 18, 4, 36-48.

Deleuze, G., Guattari, F. (1991/2005). Qu'est-ce que la philosophie, Paris : Les éditions de Minuit.

Deleuze, G. (1984). Cinéma et Pensée cours 67 du 30/10/1984 -2, consulté le 21 septembre 2018 à http://www2.univ-paris8.fr/deleuze/article.php3?id_article=5

Deleuze, G. (1980). Cours sur Leibniz / Vincennes - St. Denis. 15 avril 1980, consulté à : https://www.webdeleuze.com/textes/48

Descartes, R. (1990). Les passions de l'âme. Paris: Librairie Générale Française.

Dewey, J. (1916). Democracy and Education: An introduction to the philosophy of education, New York: The Macmillan Company.

Duclos, A.-M. (2009). Le programme de Philosophie pour enfants en contexte scolaire comme outil de compréhension des émotions chez les enfants de 5 ans. Mémoire de maîtrise. Université du Québec à Montréal.

Fiema, G. (2014). Étude des mouvements de pensée collective lors des ateliers philosophiques au primaire et au collège : Extraction de philosophèmes en tant que structures formelles de raisonnement (Thèse de Doctorat, Université Blaise Pascal Clermont-Ferrand II).

Consulté à l'adresse https://tel.archives-ouvertes.fr/tel-

01343431 
Foucault, M. (1985). "La vie : l'expérience et la science ", Revue de métaphysique et de morale, $90^{\mathrm{e}}$ année, $\mathrm{n}^{\circ} \mathrm{I}$ : Canguilhem, janvier-mars 1985, 3-14. Consulté en ligne sur http://llibertaire.free.fr/MFoucault237.html

Fournel, A. (2018). Analyse pragmatique et actionnelle de l'acte de questionner. Le questionnement chez des élèves de primaire et de collège pratiquant la philosophie à l'école (Thèse de Doctorat, Université Grenoble Alpes). Consulté à l'adresse https://tel.archives-ouvertes.fr/tel-01841459/document

Fournel, A. (à paraître). La verbalisation du désir de savoir. In Philosopher avec les enfants : fabrique de l'apprendre, fabrique du savoir, Clermont-Ferrand : Presses Universitaires Blaise Pascal.

Frege, G. (1879-1925/1994). Ecrits logiques et philosophiques. Paris : Editions du Seuil.

Giménez-Dasi, M., Daniel, M.-F., (2011). La philosophie pour enfants : l'exemple du programme "Réfléchir sur ses émotions", Diotime [en ligne], n049 (07/2011).

Hume, D. (1739/1991). Traité de la nature humaine. Livre II. Des Passions. Et Dissertation sur les passions. Trad. J.-P. Cléro. Paris : Flammarion.

Ladrière, J. (2002). " CONCEPT », In Encyclopadia Universalis, Corpus 6, Paris : Encyclopadia Universalis France.

Lagrange-Lanaspre, S. (à paraître). Figures d'analogies verbo-gestuelles et raisonnement collectif. In Philosopher avec les enfants : fabrique de l'apprendre, fabrique du savoir, Clermont-Ferrand : Presses Universitaires Blaise Pascal.

Leleux, C. (2009). La discussion à visée philosophique pour développer le jugement moral et citoyen ? Revue française de pédagogie, 166, 71-87, https://journals.openedition.org/rfp/1271

Lévine, J., Chambard, G., Sillam, M., \& Gostain, D. (2014). L'enfant philosophe, avenir de I'humanité ? Issy-les-Moulineaux: ESF Editeur.

Lipman, M. (2006). A l'école de la pensée: enseigner une pensée holistique (N. Decostre, trad.). Bruxelles, Belgique : De Boeck Université.

Lipman, M. (1991). Thinking in Education. New York: Cambridge University Press.

Lipman, M. (1989). Pixie (A. Richard, trad.). Moncton, N.-B., Canada: Éditions d'Acadie.

Lipman, M., Sharp, A. (1986). Wondering at the world: instructional manual to accompagny Kio \& Gus. Lanham, MD : University Press of America.

Nussbaum, M. C. (2004). Emotions as Judgments of Value and Importance. In R. C. Solomon (Éd.), Thinking About Feeling: Contemporary Philosophers on Emotions (183-199). Oxford: Oxford University Press.

Peirce, C. S. (1877). The Fixation of Belief, Popular Science Mounthly 12 (November), 1-15.

Polo, C. (à paraître). Les gestes métaphoriques comme construction collective d'analogies cognitives. In Philosopher avec les enfants : fabrique de l'apprendre, fabrique du savoir, Clermont-Ferrand : Presses Universitaires Blaise Pascal.

Popper K. (1960). On the Sources of Knowledge and of Ignorance. Dans Popper, K. Conjectures and Refutations, Londra, Routledge and Kegan Paul. Lu en roumain dans le recueil Filozoful rege, (1992) Bucarest: Humanitas.

Roine, P. (2016). Etude des éléments dialogiques présents dans les processus de conceptualisation lors des discussions à visée philosophique en cycle 3 de l'école élémentaire. Consulté à l'adresse https://tel.archives-ouvertes.fr/tel-01468211

Sartre, J.-P. (1938/1995). Esquisse d'une théorie des émotions. Paris : Le Livre de Poche.

Sasso, R., \& Villani, A. (2003). Le Vocabulaire de Gilles Deleuze. Paris : Vrin. 
Schleifer, M., Daniel, M.-F., Peyronnet, E., \& Lecomte, S. (2003). The Impact of Philosophical Discussions on Moral Autonomy, Judgment, Empathy and the Recognition of Emotion in Five Year Olds. Thinking, 16(4), 4-12.

Tozzi, M. (2007). Quelles compétences développent les discussions à visée philosophique (DVP) chez les élèves? Diotime [en ligne], (33). Consulté à l'adresse http://www.educrevues.fr/Diotime/affichagedocument.aspx?iddoc=32829\&pos=281

Tozzi, M. (1993). Contribution à l'élaboration d'une didactique de l'apprentissage du philosopher. Revue française de pédagogie, 103(1), 19-31. Consulté à : https://doi.org/10.3406/rfp.1993.1294

Varela, F. J., Thompson, E., \& Rosch, E. (1991). The embodied mind: cognitive science and human experience. Cambridge, Mass: MIT Press.

Vygotski, L. S. (1985). Pensée et langage (F. Sève, trad.). Paris, France: Messidor : Éditions sociales. 